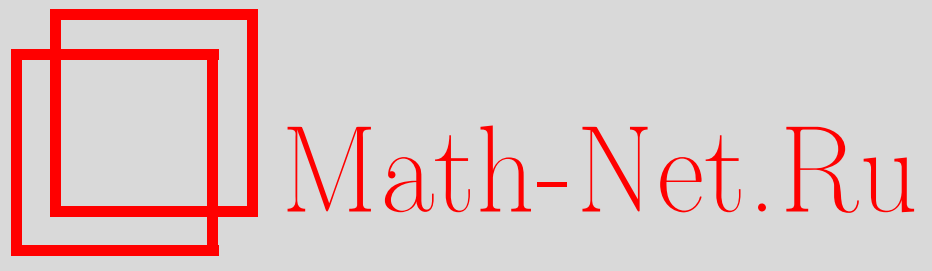

P. Del Moral, M. Doisy, Maslov idempotent probability calculus. II, Теория вероятн. и ее примен., 1999, том 44, выпуск $2,384-400$

DOI: https://doi.org/10.4213/tvp774

Использование Общероссийского математического портала Math-Net.Ru подразумевает, что вы прочитали и согласны с пользовательским соглашением http://www . mathnet.ru/rus/agreement

Параметры загрузки:

IP : 54.80 .73 .141

26 апреля 2023 г., $17: 38: 46$

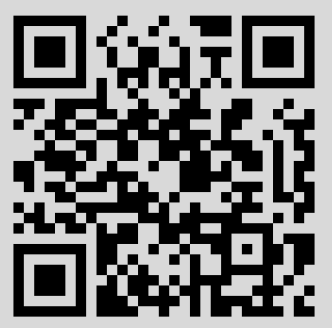


(c) $1999 \mathrm{r}$.

\author{
DEL MORAL P.*, DOISY M.**
}

\title{
MASLOV IDEMPOTENT PROBABILITY CALCULUS. II ${ }^{1)}$
}

\begin{abstract}
Изучение процессов Беллмана-Маслова привело к новому пониманию задач оптимального управления и их связи с изучением дифференциальных уравнений Гамильтона-Якоби. Цель настоящей работы - показать, что идемпотентный анализ дает естественный и общий вероятностный круг идей для изучения таких уравнений. Некоторые новые результаты, связанные с долговременным поведением решения одного класса дифференциальных уравнений Гамильтона-Якоби, могут рассматриваться как $(\max ,+)$-версия закона больших чисел и центральной предельной теоремы. Обсуждаются также применения к эволюционным уравнениям, возникающим в математической морфологии.
\end{abstract}

Клячевые слова и фразы: процессы Беллмана-Маслова, уравнения Гамильтона-Якоби, идемпотентный анализ, математическая морфология.

Introduction. Maslov Idempotent Probability Calculus gives a natural framework for formulating and studying non-linear optimal control problems and Hamilton-Jacobi equations. The evolution of this rapidly developing area of research can be seen quite directly through the following chain of papers [1]-[3], [12], [14]-[16]. The paper [24] also contains a useful survey on this subject. Using this framework we have shown in the first part of this paper that the Bellman optimality principle may be viewed as a basic definition of a class of processes like Markov's property rather than a conclusion. We will not repeat the basic concepts such as performance spaces or independence and conditioning. One can also find there a list of references.

The study of Bellman-Maslov processes has lead to new advances in the understanding of non-linear optimal control problems and in the study of fixed points of non-expansive transformations. In [11] we proved that the

${ }^{*}$ LSP-UMR C55830, CNRS, Bat. 1R1, Univ. Paul Sabatier, 31062 Toulouse, France; e-mail: delmoral@cict.fr

${ }^{* *}$ ENSEEIHT, 2 Rue Camichel, 31071 Toulouse, and LSP-UMR C55830, CNRS, France; e-mail: doisy@len7.enseeiht.fr

1) This work was supported by the INTAS-RFBR grant 95-91. 
Hamiltonian operator can be regarded as the $(\max ,+)$-version of the classical Kolmogorov differential operator of Stochastic Calculus. Furthermore some new stability properties of Bellman-Maslov processes have been obtained using the $(\max ,+)$-version of the celebrated Dynkin's formula in stochastic process theory.

Using this framework the fixed points of non-expansive transformations are the $(\max ,+)$-version of the invariant probability measures for discrete time Markov semi-groups. Applications to generalized simulated annealing and genetic type algorithms are given in [11] (see also [17] for a detailed study of the convergence of such stochastic algorithms).

In the present work the idempotent probability calculus presented in the first part is applied to study a class of Hamilton-Jacobi equations arising in optimal control problems and in Mathematical Morphology. The paper has the following structure.

In the first section we extend the analysis of Hamilton-Jacobi equations presented in [22] and [23] to more general classes of Hamilton-Jacobi equations and we connect these new generalized solutions with the classical viscosity solutions (see [4], [8], [9] and references therein). What also makes our result interesting and new is that we propose a natural and general «probabilistic line of thought» to study these Hamilton-Jacobi equations. Using $(\max ,+)$-semigroup technics we will prove that the so-called Maslov generalized solution of a first order Hamilton-Jacobi differential equation coincides with the known classical viscosity solution. We will give a complete proof of this statement and explain its relevance to obtain explicit solutions such as the Oleinik-Lax formula and to study evolution equations arising in Mathematical Morphology.

In Section 2 we study the limiting behavior of a class of HamiltonJacobi equations. The weak law of large numbers and the central limit theorem presented in this section are the time continuous version of those presented in [30] and [12].

In the last section we use the preceding framework to make sense out of the evolution equations arising in Mathematical Morphology. This new application of idempotent analysis provides an alternative approach to the Beucher's morphological gradient or the sup-inf derivative notions presented in $[6],[31]$.

1. Generalized solution of Hamilton-Jacobi equations. Using Maslov's optimization framework we discuss now the solution of first order Hamilton-Jacobi equations. This part of the paper essentially records works of Kolokoltsov and Maslov [22]. In [22] the authors make use of idempotent analysis to propose a generalized solution of a class of Hamilton-Jacobi equations. Our main contribution is to extend their result to more general classes of Hamilton-Jacobi equations and to connect these new generalized 
solutions with the classical viscosity solutions (see [4], [8], [9] and references therein). As in [4] the main tools here are the so-called relaxed controls.

The class of differential equations studied in this paper has already been studied in details in several works (see for instance [4]). What makes our result interesting and new is that we propose a natural and general «probabilistic line of thought» to study these Hamilton-Jacobi equations. All the methods of proof we describe in this paper are built upon the application of the idempotent analysis framework developed in the first part of this study. This will provide a good illustration of the application of Maslov's optimization theory. A practical advantage of this approach is that it leads to a new understanding of such equations. For instance we will see that the Hamiltonian operator is the $(\max ,+)$-version of the Kolmogorov differential operator associated with a Markov diffusion process. On the other hand the viscosity solution of an Hamilton-Jacobi equation with discontinuous initial value coincides with the weak solution in idempotent analysis framework.

Let $U$ be a compact metric space. By $\mathbf{M}_{1}(U)$ we denote the space of all probability measures on $U$ furnished with the weak ${ }^{\star}$ topology. Let us assume that $\Omega=L^{\infty}\left((0,1), \mathbf{M}_{1}(U)\right)$ is equipped with the weak ${ }^{\star}$ topology. We recall that $\mu^{n}$ converges weakly to $\mu$ as $n \rightarrow \infty$ if and only if for any continuous function $\phi:(0,1) \times U \longrightarrow \mathbf{R}$

$$
\lim _{n \rightarrow \infty} \int_{(0,1) \times U} \phi(t, u) \mu_{t}^{n}(d u) d t=\int_{(0,1) \times U} \phi(t, u) \mu_{t}(d u) d t .
$$

For this topology, $L^{\infty}\left((0,1), \mathbf{M}_{1}(U)\right)$ is a compact space and the points of this set as usually are called relaxed controls.

Let $\mathscr{C}\left((0,1), \mathbf{R}^{d}\right), d \geqslant 1$, be the space of all continuous mappings furnished with the uniform norm. For any $x \in \mathbf{R}^{d}$ we define $X^{x}: \omega \in$ $\Omega \longrightarrow X^{x}(\omega) \in \mathscr{C}\left((0,1), \mathbf{R}^{d}\right)$ by setting

$$
X_{t}^{x}(\omega)=x+\int_{0}^{t} F\left(X_{s}^{x}(\omega), \omega_{s}\right) d s, \quad F(x, \mu)=\int_{U} f(x, u) \mu(d u),
$$

where $f: \mathbf{R}^{d} \times U \longrightarrow \mathbf{R}^{d}$ is a bounded continuous function so that $\mid f(x, u)-$ $f(y, u)|\leqslant C| x-y \mid$. In this framework the path $t \in(0,1) \longrightarrow X_{t}^{x}(\omega) \in \mathbf{R}^{d}$ is called the relaxed path associated with $\omega$ with initial condition $x \in \mathbf{R}^{d}$. One can check that $X^{x}$ is continuous and therefore it can always be regarded as an optimization variable taking values in $\mathscr{C}\left((0,1), \mathbf{R}^{d}\right)$.

Now, let $\mathbf{p}_{x}: \Omega \rightarrow \mathbf{R}_{\max }$ be defined as

$$
\mathbf{p}_{x}(\omega)=\int_{0}^{1} L\left(X_{t}^{x}(\omega), \omega_{t}\right) d t, \quad L(x, \mu)=\int_{U} l(x, u) \mu(d u),
$$

where $l: \mathbf{R}^{d} \times U \longrightarrow \mathbf{R}_{\max }$ is a bounded continuous function so that $\mid l(x, u)-$ $l(z, u)|\leqslant C| x-z \mid$. 
We will always assume that the following condition holds

(H0) for any $x \in \mathbf{R}^{d}, s \leqslant t$, there exists a control $\omega \in \Omega$ such that $\int_{s}^{t} L\left(X_{r}^{s, x}(\omega), \omega_{r}\right) d r=0$, where $\left\{X_{r}^{s, x}(\omega): r \in[s, t]\right\}$ is the solution of (1) such that $X_{s}^{s, x}(\omega)=x$ and for any performance density $\mu$ on $\mathbf{R}^{d}$ the mapping

$$
(x, \omega) \in \mathbf{R}^{d} \times \Omega \longrightarrow \mu(x)+\int_{0}^{1} L\left(X_{t}^{x}(\omega), \omega_{t}\right) d t \in \mathbf{R}_{\max }
$$

is a well-defined performance density on $\mathbf{R}^{d} \times \Omega$. Under our assumptions we have that:

- For any $x \in \mathbf{R}^{d}$ there exists some $\omega_{x} \in \Omega$ such that $\mathbf{p}_{x}\left(\omega_{x}\right)=0$ and therefore $\mathbf{P}_{x}(\Omega)=\mathbb{I}$

- For any $x \in \mathbf{R}^{d}\left(\Omega, \mathscr{B}(\Omega), \mathbf{P}_{x}, X^{x}\right)$ is a well-defined Bellman-Maslov process and the conditional density of $X_{t}$ given $X_{s}, s \leqslant t$, is given by the formula

$$
\mathbf{p}_{t \mid s}^{X}(y \mid z)=\sup \left\{\int_{s}^{t} L\left(X_{r}^{s, z}(\omega), \omega_{r}\right) d r ; \omega \in \Omega: X_{t}^{s, z}(\omega)=y\right\},
$$

where $\left\{X_{r}^{s, z}(\omega): r \in[s, t]\right\}$ is the solution of (1) such that $X_{s}^{s, z}(\omega)=z$.

- For any performance density $\mu$ on $\mathbf{R}^{d}$ the mapping

$$
x \in \mathbf{R}^{d} \longrightarrow \int^{\oplus} \mathbf{p}_{t \mid 0}(x \mid z) \odot \mu(z) \odot d z \in \mathbf{R}_{\max }
$$

is a performance measure on $\mathbf{R}^{d}$.

One clear consequence of Proposition 1 in the first part of this study is that the covariant measure $\mathbf{P}_{x}^{X}=\mathbf{P}_{x} \circ\left(X^{x}\right)^{-1}$ of $\mathbf{P}_{x}$ under the optimization variable $X^{x}$ is a performance measure with density

$$
\mathbf{p}_{x}^{X}(z)=\sup \left\{\int_{0}^{1} L\left(X_{t}^{x}(\omega), \omega_{t}\right) d t ; \omega \in L^{\infty}\left((0,1), \mathbf{M}_{1}(U)\right): X^{x}(\omega)=z\right\} .
$$

By definition of the optimization process $X$ we have that $\mathbf{p}_{x}^{X}(z)=-\infty=0$ whenever $z_{0} \neq x_{0}$. We will denote by $\mathbf{E}_{x}(\cdot)$ the $(\max ,+)$-expectations with respect to the performance measure $\mathbf{P}_{\boldsymbol{x}}$.

In order to carry our analysis to the next stage it is convenient to recall some classical results on relaxed controls. As usually we associate with each «classical» control $u(\cdot) \in L^{\infty}((0,1), U)$ a relaxed control $t \longrightarrow \delta_{u(t)} \in L^{\infty}\left((0,1), \mathbf{M}_{1}(U)\right)$. Using this identification it is well known that $L^{\infty}((0,1), U)$ is a dense subset of $\Omega=L^{\infty}\left((0,1), \mathbf{M}_{1}(U)\right)$. Then, using the fact that the density $\mathbf{p}$ is continuous one concludes that $\forall x \in \mathbf{R}^{d}$, $\forall A \in \mathscr{B}(\Omega)$

$$
\begin{aligned}
\mathbf{P}_{x}(A) & =\mathbf{P}_{x}\left(A \cap L^{\infty}((0,1), U)\right) \\
& =\sup \left\{\int_{0}^{1} l\left(X_{t}^{x}(u), u_{t}\right) d t ; \quad u \in A \cap L^{\infty}((0,1), U)\right\} .
\end{aligned}
$$


Note that the above result remains valid if the density $\mathbf{p}$ is only uppersemicontinuous on $\Omega$.

It is now convenient to introduce the Hamilton-Jacobi operator associated with the time-homogeneous Bellman-Maslov process $X$.

Definition-Proposition 1. For any $x \in \mathbf{R}^{d}$ and $p \in \mathbf{R}^{d}$ we have

$$
\lim _{h \rightarrow 0} h^{-1} \mathbf{E}_{x}\left(\left\langle p, X_{h}^{x}-x\right\rangle\right)=H(x, p),
$$

where $H: \mathbf{R}^{d} \times \mathbf{R}^{d} \longrightarrow \mathbf{R}_{\max }$ is the Hamiltonian function associated with $l$ and given by

$$
H(x, p)=\sup _{u \in U}(\langle p, f(x, u)\rangle+l(x, u)) .
$$

P r o of. We first notice that

$$
\begin{aligned}
h^{-1} \mathbf{E}_{x}\left(\left\langle p, X_{h}^{x}-x\right\rangle\right) & =\sup \left\{\left\langle p, h^{-1}\left(X_{h}^{x}-x\right)\right\rangle+h^{-1} \int_{0}^{h} l\left(X_{t}^{x}(u), u_{t}\right) d t\right\} \\
& =\sup \left\{h^{-1} \int_{0}^{h}\left[\left\langle p, f\left(X_{t}^{x}, u_{t}\right)\right\rangle+l\left(X_{t}^{x}(u), u_{t}\right)\right] d t\right\},
\end{aligned}
$$

where the supremum run over all «classical» controls $u \in L^{\infty}((0,1), U)$ and the path $\left\{X_{t}^{x}(u) ; 0 \leqslant t \leqslant h\right\}$ is the solution of (1) associated with $u(\cdot)$ and such that $X_{0}^{x}(u)=x$. Taking a constant control $u(\cdot)=v \in U$ and letting $h \rightarrow 0$ we clearly have

$$
\lim _{h \rightarrow 0} h^{-1} \mathbf{E}_{x}\left(\left\langle p, X_{h}^{x}-x\right\rangle\right) \geqslant\langle p, f(x, v)\rangle+l(x, v) \quad \forall v \in U .
$$

Hence, $\lim _{h \rightarrow 0} h^{-1} \mathbf{E}_{x}\left(\left\langle p, X_{h}^{x}-x\right\rangle\right) \geqslant H(x, p)$. On the other hand, the Lipschitz assumptions on the mappings $l$ and $f$ yield

$\left\langle p, f\left(X_{t}^{x}, u_{t}\right)\right\rangle+l\left(X_{t}^{x}(u), u_{t}\right) \leqslant\left\langle p, f\left(x, u_{t}\right)\right\rangle+l\left(x, u_{t}\right)+$ const $\left\|X_{t}^{x}(u)-x\right\|$.

This implies that

$$
h^{-1} \int_{0}^{h}\left[\left\langle p, f\left(X_{t}^{x}, u_{t}\right)\right\rangle+l\left(X_{t}^{x}(u), u_{t}\right)\right] d t \leqslant H(x, p)+\text { const }\|f\| h
$$

and therefore $\lim _{h \rightarrow 0} h^{-1} \mathbf{E}_{x}\left(\left\langle p, X_{h}^{x}-x\right\rangle\right) \leqslant H(x, p)$.

Let us recall the notion of continuous super-solution and sub-solution of the Hamilton-Jacobi equation

$$
\frac{\partial \phi}{\partial t}=H\left(x, D_{x} \phi\right) .
$$

The main difficulty in the study of these equations comes from the fact that their solution $(t, x) \rightarrow \phi(t, x)$ may fail to be differentiable at each point $(t, x)$. 
It is therefore natural to introduce the notion of super-gradient $D^{+} \phi\left(t_{0}, x_{0}\right)$ and sub-gradient $D^{-} \phi\left(t_{0}, x_{0}\right)$ of a continuous function $\phi$ at $\left(t_{0}, x_{0}\right)$ :

$$
\begin{aligned}
D^{+} \phi\left(t_{0}, x_{0}\right)= & \left\{(p, q) \in \mathbf{R} \times \mathbf{R}^{d} ;\right. \\
& \left.\limsup _{(t, x) \rightarrow\left(t_{0}, x_{0}\right)} \frac{\phi(t, x)-\phi\left(t_{0}, x_{0}\right)-p\left(t-t_{0}\right)-q \cdot\left(x-x_{0}\right)}{\left(\left|t-t_{0}\right|^{2}+\left|x-x_{0}\right|^{2}\right)^{1 / 2}} \leqslant 0\right\}, \\
D^{-} \phi\left(t_{0}, x_{0}\right)= & \left\{(p, q) \in \mathbf{R} \times \mathbf{R}^{d} ;\right. \\
& \left.\liminf _{(t, x) \rightarrow\left(t_{0}, x_{0}\right)} \frac{\phi(t, x)-\phi\left(t_{0}, x_{0}\right)-p\left(t-t_{0}\right)-q \cdot\left(x-x_{0}\right)}{\left(\left|t-t_{0}\right|^{2}+\left|x-x_{0}\right|^{2}\right)^{1 / 2}} \geqslant 0\right\} .
\end{aligned}
$$

Defin ition 1. A continuous function $\phi$ such that

$$
\forall\left(t_{0}, x_{0}\right) \quad \forall(p, q) \in D^{+} \phi\left(t_{0}, x_{0}\right) \quad H\left(x_{0}, q\right) \geqslant p
$$

is called a super-solution of the Hamilton-Jacobi equation (3). We say that $\phi$ is a sub-solution of (3) when

$$
\forall\left(t_{0}, x_{0}\right) \quad \forall(p, q) \in D^{-} \phi\left(t_{0}, x_{0}\right) \quad H\left(x_{0}, q\right) \leqslant p .
$$

$\phi$ is a solution of (3) if it is both a super-solution and a sub-solution.

Theorem 1. For any $\varphi \in \operatorname{Lip}\left(\mathbf{R}^{d}\right)$ the mapping $\phi(t, x)=\mathbf{P}_{t} \varphi(x) \stackrel{\text { def }}{=}$ $\mathrm{E}\left(\varphi\left(X_{t}^{x}\right)\right)$ belongs to $\operatorname{Lip}\left((0,1) \times \mathbf{R}^{d}\right)$ and it is the unique bounded and uniformly continuous solution of the Hamilton-Jacobi equation

$$
\left\{\begin{array}{l}
\frac{\partial \phi}{\partial t}=H\left(x, D_{x} \phi\right), \\
\phi(0, \cdot)=\varphi(\cdot) .
\end{array}\right.
$$

In addition $\left\{\mathbf{P}_{t} ; t \geqslant 0\right\}$ is a monotone semi-group of $(\oplus, \odot)$-linear operators on $\operatorname{Lip}\left(\mathbf{R}^{d}\right)$ :

1) $\forall 0 \leqslant s \leqslant t \leqslant 1 \mathbf{P}_{t} \circ \mathbf{P}_{s}=\mathbf{P}_{t+s}, \mathbf{P}_{0}=\mathrm{Id}$

2) $\varphi_{1} \leqslant \varphi_{2} \Longrightarrow \mathbf{P}_{t} \varphi_{1} \leqslant \mathbf{P}_{t} \varphi_{2} \forall t \in[0,1]$;

3) for any $a_{1}, a_{2} \in \mathbf{R}_{\max }$ and $\varphi_{1}, \varphi_{2} \in \operatorname{Lip}\left(\mathbf{R}^{d}\right)$ and $t \in[0,1]$

$$
\mathbf{P}_{t}\left(a_{1} \odot \varphi_{1} \oplus a_{2} \odot \varphi_{2}\right)=a_{1} \odot \mathbf{P}_{t}\left(\varphi_{1}\right) \oplus a_{2} \odot \mathbf{P}_{t}\left(\varphi_{2}\right) .
$$

$\mathrm{P} \mathrm{r}$ o of. The proof of the theorem is essentially contained in [4]. Since the initial condition is Lipschitz one the uniqueness of the solution in the class of bounded and uniformly continuous functions is a well known result. We now show that the tools presented before induce a simple proof of this theorem. 
To prove that $\phi \in \operatorname{Lip}\left((0,1) \times \mathbf{R}^{d}\right)$ we use the fact that for any $\omega, \omega^{\prime} \in \Omega$ and $x, y \in \mathbf{R}^{d}$

$$
\left|X_{t}^{x}(\omega)-X_{t}^{y}\left(\omega^{\prime}\right)\right| \leqslant \text { const }|x-y| .
$$

The proof of this inequality is standard and it is a consequence of Gronwall's lemma. Using the above observation (2) and the classical inequality $\sup \{(1)\}-\sup \{(2)\} \leqslant \sup \{(1)-(2)\}$ one obtain easily for any $s \leqslant t$

$$
\begin{aligned}
& \mathbf{P}_{t}(\varphi)(x)-\mathbf{P}_{s}(\varphi)(y) \\
& \quad \leqslant \sup \left\{\int_{0}^{t} l\left(X_{r}^{x}(u), u_{\tau}\right) d r-\int_{0}^{s} l\left(X_{r}^{y}(u), u_{r}\right) d r+\varphi\left(X_{t}^{x}(u)\right)-\varphi\left(X_{s}^{y}(u)\right)\right\}
\end{aligned}
$$

where the supremum runs over all $u \in L^{\infty}((0,1), U)$. Under our Lipschitz assumptions on $l$ and $\varphi$ this implies that

$$
\begin{aligned}
& \mathbf{P}_{t}(\varphi)(x)-\mathbf{P}_{s}(\varphi)(y) \\
& \quad \leqslant \text { const }\left(|t-s|+\int_{0}^{s}\left\|X_{r}^{x}(u)-X_{r}^{y}(u)\right\| d r+\left\|X_{t}^{x}(u)-X_{t}^{y}(u)\right\|\right) .
\end{aligned}
$$

Combining the above inequality with (5) we conclude that

$$
\mathbf{P}_{t}(\varphi)(x)-\mathbf{P}_{s}(\varphi)(y) \leqslant \text { const }(|t-s|+|x-y|) .
$$

This proves that $\phi \in \operatorname{Lip}\left((0,1) \times \mathbf{R}^{d}\right)$.

To prove that $\left\{\mathbf{P}_{t} ; t \geqslant 0\right\}$ is a monotone semi-group of $(\oplus, \odot)$-linear operators on $\operatorname{Lip}\left(\mathbf{R}^{d}\right)$ it suffices to note that

$$
\begin{aligned}
\mathbf{P}_{t+s}(\varphi)(x) & =\mathbf{E}\left(\varphi\left(X_{t+s}^{x}\right)\right)=\mathbf{E}\left(\mathbf{E}\left(\varphi\left(X_{t+s}^{x}\right) \mid X_{s}^{x}\right)\right) \\
& =\mathbf{E}\left(\mathbf{P}_{t}\left(X_{s}^{x}\right)\right)=\mathbf{P}_{s}\left(\mathbf{P}_{t} \varphi(x)\right) .
\end{aligned}
$$

To end the proof of the theorem it remains to check that $\phi(t, x)=\mathbf{P}_{t}(\varphi)(x)$ is solution of (4). First we note that

$$
\begin{aligned}
(p, q) \in D^{+} \phi(t, x) & \Longrightarrow \phi\left(t-h, X_{h}^{x}\right)-\phi(t, x) \\
& \leqslant-p h+q \cdot\left(X_{h}^{x}-x\right)+o(h), \\
\left(p^{\prime}, q^{\prime}\right) \in D^{-} \phi(t, x) & \Longrightarrow \phi\left(t-h, X_{h}^{x}\right)-\phi(t, x) \\
& \geqslant-p^{\prime} h+q^{\prime} \cdot\left(X_{h}^{x}-x\right)+o(h) .
\end{aligned}
$$

On the other hand we have

$$
\phi(t, x)=\mathbf{E}\left(\mathbf{E}\left(\varphi\left(X_{t}^{x}\right) \mid X_{h}^{x}\right)\right)=\mathbf{E}\left(\phi\left(t-h, X_{h}^{x}\right)\right) .
$$

Hence, $\mathbf{E}\left(\phi\left(t-h, X_{h}^{x}\right)-\phi(t, x)\right)=0$. By definition of the super-gradient $(p, q) \in D^{+} \phi(t, x)$ and sub-gradient $\left(p^{\prime}, q^{\prime}\right) \in D^{-} \phi(t, x)$ one gets

$$
h^{-1} \mathbf{E}_{x}\left(\left\langle q, X_{h}^{x}-x\right\rangle\right) \geqslant p+O(h), \quad h^{-1} \mathbf{E}_{x}\left(\left\langle q^{\prime}, X_{h}^{x}-x\right\rangle\right) \leqslant p^{\prime}+O(h) .
$$


Letting $h \rightarrow 0$ and using Proposition 1 one concludes that $H(x, q) \geqslant p$, $H\left(x, q^{\prime}\right) \leqslant p^{\prime}$ and the proof of the theorem is completed.

By Rademacher's theorem every Lipschitz function is differentiable at almost all points $(t, x)$. In view of the proof of the theorem, if $\phi$ is differentiable at $(t, x)$ then we have

$$
h^{-1} \mathbf{E}_{x}\left(\left\langle D_{x} \phi(t, x), X_{h}^{x}-x\right\rangle\right)=\frac{\partial \phi}{\partial t}(t, x)+O(h) .
$$

Letting $h \rightarrow 0$ it follows that $(t, x) \longrightarrow \phi(t, x)=\mathbf{P}_{t} \varphi(x)$ satisfies (3) for almost all $(t, x)$.

In order to point out the connections between these results and the classical viscosity solution we recall that the super-gradient and the subgradient of a continuous function $\phi$ can also be defined as follows

$$
\begin{aligned}
D^{+} \phi(t, x)= & \left\{D \psi(t, x) ; \psi \in \mathscr{C}^{1}\left((0,1) \times \mathbf{R}^{d}\right):\right. \\
(t, x) \text { is a local maxima of } \phi-\psi\}, & \left\{D \psi(t, x) ; \psi \in \mathscr{C}^{1}\left((0,1) \times \mathbf{R}^{d}\right):\right. \\
D^{-} \phi(t, x)= & (t, x) \text { is a local minima of } \phi-\psi\} .
\end{aligned}
$$

As a clear consequence of the above observation, $\phi$ is a super-solution of (3) if and only if for any $(t, x)$ and $\psi \in \mathscr{C}^{1}$ such that $(t, x)$ is a local maxima of $\phi-\psi$

$$
H\left(x, D_{x} \psi(t, x)\right) \geqslant \frac{\partial \psi}{\partial t}(t, x) .
$$

If $\psi \in \mathscr{C}^{1}$ and $(t, x)$ is a local maximum of $\phi-\psi$ then there exists an open ball $B((t, x), r), r>0$, such that

$$
\phi(s, y)-\psi(s, y) \leqslant \phi(t, x)-\psi(t, x) \quad \forall(s, y) \in B((t, x), r) .
$$

Since

$$
\psi(s, y)=\psi(t, x)+\frac{\partial \psi}{\partial t}(t, x)(s-t)+D_{x} \psi(t, x)(y-x)+o(|t-s|+|y-x|)
$$

letting $s=t-h$ and $y=X_{h}^{x}(u), u \in L^{\infty}$, this implies that

$$
\phi\left(t-h, X_{h}^{x}(u)\right)-\phi(t, x) \leqslant \frac{\partial \psi}{\partial t}(t, x)(-h)+D_{x} \psi(t, x)\left(X_{h}^{x}(u)-x\right)+o(h)
$$

for sufficiently small $h>0$. Using the same line of arguments as before one concludes that

$$
H\left(x, D_{x} \psi(t, x)\right) \geqslant \frac{\partial \psi}{\partial t}(t, x)
$$

If the initial value condition of an Hamilton-Jacobi equation is not sufficiently regular Theorem 1 is not applicable. More precisely solutions of Hamilton-Jacobi equations with discontinuous initial value are not always 
continuous and so we should not be able to apply the approach presented above.

In order to carry our analysis to the next stage we note that the transition performance densities

$$
\mathbf{p}_{t \mid 0}^{X}(z \mid x)=\sup \left\{\int_{s}^{t} l\left(X_{r}^{x}(u), u_{r}\right) d r ; u \in L^{\infty}: X_{t}^{x}(u)=z\right\}
$$

generate two optimization operations. The first one acting on Lipschitz continuous function $\varphi$ and the second one acting on performance densities $\mu$ :

$$
\begin{aligned}
& \mathbf{P}_{t}(\varphi)(x)=\int^{\oplus} \varphi(z) \odot \mathbf{p}_{t \mid 0}^{X}(z \mid x) \odot d z, \\
& \left(\mu \mathbf{P}_{t}\right)(z)=\int^{\oplus} \mathbf{p}_{t \mid 0}^{X}(z \mid x) \odot \mu(x) \odot d z .
\end{aligned}
$$

We have already proved that the first operation gives a complete answer to the Hamilton-Jacobi problem (4) with initial value $\varphi$. The second operation can also be rewritten using the duality relation

$$
\left\langle\mu, \mathbf{P}_{t} \varphi\right\rangle=\left\langle\mu \mathbf{P}_{t}, \varphi\right\rangle \quad \forall \varphi \in \operatorname{Lip}\left(\mathbf{R}^{d}\right) .
$$

Now let us assume that $\mu \in \operatorname{Lip}\left(\mathbf{R}^{r}\right)$. In this situation we have

$$
\mu \mathbf{P}_{t}(x)=\sup \left\{\mu(z)+\int_{0}^{t} l\left(X_{s}^{z}(u), u_{s}\right) d s ; u \in L^{\infty}: X_{t}^{z}(u)=x\right\}
$$

and therefore

$$
\mu \mathbf{P}_{t}(x)=\sup \left\{\int_{0}^{t} l\left(\tilde{X}_{s}^{x}(u), u_{s}\right) d s+\mu\left(\tilde{X}_{t}^{x}(u)\right) ; u \in L^{\infty}\right\},
$$

where $\forall 0 \leqslant s \leqslant t, \dot{\tilde{X}}_{s}^{x}(u)=-f\left(\tilde{X}_{s}^{x}(u), u_{s}\right), \tilde{X}_{0}^{x}(u)=x$. Theorem 1 clearly implies that $m(t, x)=\mu \mathbf{P}_{t}(x)$ is the unique bounded and uniformly continuous solution of the dual Hamilton-Jacobi equation

$$
\left\{\begin{array}{l}
\frac{\partial m}{\partial t}=H^{(\star)}\left(x, D_{x} m\right), \\
m(0, \cdot)=\mu(\cdot)
\end{array}\right.
$$

where $H^{(*)}(x, p)=\sup _{u \in U}(\langle p,-f(x, u)\rangle+l(x, u))=H(x,-p)$.

Definition 2. For any performance density $\mu$ on $\mathbf{R}^{d}$ a mapping $(t, x) \longrightarrow m_{t}(x) \in \mathbf{R}_{\max }$ such that for any $t \in(0,1) m_{t}$ is a performance density on $\mathbf{R}^{d}$ and $m_{0}(x)=\mu(x)$ and

$$
\forall \varphi \in \operatorname{Lip}\left(\mathbf{R}^{d}\right) \quad\left\langle\varphi, m_{t}\right\rangle=\left\langle\mu, \mathbf{P}_{t} \varphi\right\rangle
$$

is called a weak solution of the dual Hamilton-Jacobi equation (6).

The above definition and Theorem 1 and Kolokoltsov-Maslov Theorem 1 in the first part of this study lead to the following 
Theorem 2. For any performance density $\mu: \mathbf{R}^{d} \longrightarrow \mathbf{R}_{\max }$ the mapping $m(t, x)=\mu \mathbf{P}_{t}$ is a performance density and it is the unique weak solution of the dual Hamilton-Jacobi equation

$$
\left\{\begin{array}{l}
\frac{\partial m}{\partial t}=H^{(\star)}\left(x, D_{x} m\right), \\
m(0, \cdot)=\mu(\cdot) .
\end{array}\right.
$$

In addition $\left\{\mathbf{P}_{t} ; t \geqslant 0\right\}$ is a monotone semi-group of $(\oplus, \odot)$-linear operators on the set of performance densities:

1) $\forall 0 \leqslant s \leqslant t \leqslant 1 \mathbf{P}_{t} \circ \mathbf{P}_{s}=\mathbf{P}_{t+s}, \mathbf{P}_{0}=\mathrm{Id}$;

2) $\mu_{1} \leqslant \mu_{2} \Longrightarrow \mu_{1} \mathbf{P}_{t} \leqslant \mu_{2} \mathbf{P}_{t} \forall t \in[0,1]$;

3) for any $a_{1}, a_{2} \in \mathbf{R}_{\max }$ such that $a_{1} \oplus a_{2}=\mathbb{I}$ and $\mu_{1}, \mu_{2}$ and $t \in[0,1]$

$$
\left(a_{1} \odot \mu_{1} \oplus a_{2} \odot \mu_{2}\right) \mathbf{P}_{t}=a_{1} \odot \mu_{1} \mathbf{P}_{t} \oplus a_{2} \odot \mu_{2} \mathbf{P}_{t} .
$$

Our next objective is to connect the notion of weak solution defined above with the classical viscosity solution of (7). We recall that a locally bounded upper-semicontinuous function $m(t, x)$ is a viscosity solution of $(7)$ whenever

1) $m$ is a super-solution of (7), i.e., for any $(t, x)$ and $\psi \in \mathscr{C}^{1}$ such that $(t, x)$ is a local maxima of $m-\psi$

$$
H^{(\star)}\left(x, D_{x} \psi(t, x)\right) \geqslant \frac{\partial \psi}{\partial t}(t, x) ;
$$

2) $m$ is a sub-solution of (7), i.e., for any $(t, x)$ and $\psi \in \mathscr{C}^{1}$ such that $(t, x)$ is a local minima of $m-\psi$

$$
H^{(\star)}\left(x, D_{x} \psi(t, x)\right) \leqslant \frac{\partial \psi}{\partial t}(t, x) .
$$

We will use the following conditions on the Hamiltonian function.

(H) $H$ is a convex function on $\mathbf{R}^{d} \times \mathbf{R}^{d}$ and a uniformly continuous function on $\mathbf{R}^{d} \times \bar{B}(0, R)$ for any $R>0$ such that

$$
\left|\frac{\partial H}{\partial x}(x, p)\right| \leqslant C(1+|p|), \quad\left|\frac{\partial H}{\partial p}(x, p)\right| \leqslant C
$$

for some finite constant $C$.

Under these assumptions for any bounded performance density $\mu$ there exists a unique upper-semicontinuous solution of (7) with initial value $\mu$ (see for instance Theorem 5.15 in [4]). Let us check that in this case $(t, x) \longrightarrow m(t, x)=\mu \mathbf{P}_{t}(x)$ is the desired solution.

Since $\mu$ is a bounded performance density there exists a non-increasing sequence of uniformly bounded Lipschitz functions $\left\{\mu^{(n)} ; n \geqslant 1\right\}$ such that $\mu=\inf _{n \geqslant 1} \mu^{(n)}$. Letting $m^{(n)}(t, x)=\mu^{(n)} \mathbf{P}_{t}(x)$ we clearly have

$$
m(t, x)=\mu \mathbf{P}_{t}(x) \leqslant \inf _{n \geqslant 1} m^{(n)}(t, x) .
$$


Now, let $\left\{\omega^{n} ; n \geqslant 1\right\}$ be a sequence of relaxed controls such that

$$
m^{(n)}(t, x)=\int_{0}^{t} L\left(\widetilde{X}_{s}^{x}\left(\omega^{n}\right), \omega_{s}^{n}\right) d s+\mu^{(n)}\left(\widetilde{X}_{t}^{x}\left(\omega^{n}\right)\right) .
$$

Under our assumptions there exists a subsequence $\left\{\omega^{n_{k}} ; k \geqslant 1\right\}$ such that $\omega^{n_{k}}$ converges to some relaxed control $\omega$ and $\widetilde{X}^{x}\left(\omega^{n_{k}}\right)$ converges to $\widetilde{X}^{x}(\omega)$ and

$$
\int_{0}^{t} L\left(\tilde{X}_{s}^{x}\left(\omega^{n_{k}}\right), \omega_{s}^{n_{k}}\right) d s \longrightarrow \int_{0}^{t} L\left(\tilde{X}_{s}^{x}(\omega), \omega_{s}\right) d s
$$

as $k \rightarrow \infty$. This yields

$$
\inf _{n \geqslant 1} m^{(n)}(t, x) \leqslant \int_{0}^{t} L\left(\widetilde{X}_{s}^{x}(\omega), \omega_{s}\right) d s+\limsup _{k \rightarrow \infty} \mu^{n_{k}}\left(\widetilde{X}_{t}^{x}\left(\omega^{n_{k}}\right)\right) .
$$

Using the fact that $\left\{\mu_{n_{k}} ; k \geqslant 1\right\}$ is a non-increasing sequence of uniformly bounded and continuous functions and $\mu$ is upper-semicontinuous one concludes that

$$
\inf _{n \geqslant 1} m^{(n)}(t, x) \leqslant \int_{0}^{t} L\left(\tilde{X}_{s}^{x}(\omega), \omega_{s}\right) d s+\mu\left(\tilde{X}_{t}^{x}(\omega)\right) .
$$

Now it is not difficult to check that

$$
m(t, x)=\mu \mathbf{P}_{t}(x)=\inf _{n \geqslant 1} m^{(n)}(t, x)=\inf _{n \geqslant 1}\left(\mu^{(n)} \mathbf{P}_{t}(x)\right) .
$$

Since each $m^{(n)}$ is solution of the Hamilton-Jacobi equation (7), $m$ is again solution of (7) (see for instance Theorems 5.13 and 5.15 in [4]).

2. Limit theorems. Our aim is now to study some limit theorems relating to the limiting behavior of the solution of the Hamilton-Jacobi equation (7) as $t \rightarrow \infty$. We will only consider a very special class of equations, the extension to more general situations is still an open subject of investigation.

The weak law of large numbers and the central limit theorem presented hereafter are the time continuous version of those presented in [30] and [12].

In a first stage we will show how the preceding results apply to obtain the so-called Oleinik-Lax explicit formula. Then we use this formula to study the limiting behavior of these explicit solutions as $t \rightarrow \infty$.

Let us suppose that the Lagrangian function $l(x, u)$ does not depend on the first parameter and it is a convex function on $U$. For any $x \in \mathbf{R}^{d}$ let $X^{x}$ be the Bellman-Maslov process defined by the equation

$$
X^{x}(u)_{t}=x+\int_{0}^{t} u_{s} d s, \quad u \in L^{\infty}((0,1) ; U)
$$

where $U$ is a compact subset of $\mathbf{R}^{d}$. We first note that in this simple example the Hamiltonian function $H(x, p)$ does not depend on the first parameter and 
it is the convex function given by

$$
H(p)=\sup _{u \in U}(\langle p, u\rangle+l(u))=(-l)^{\star}(p),
$$

where $(\cdot)^{\star}$ stands for the Fenchel transform of a convex function. By the involutive property of the Fenchel transform we also have $H^{\star}=(-l)^{\star \star}=-l$. Then, for any $\varphi \in \operatorname{Lip}\left((0,1) \times \mathbf{R}^{d}\right)$ the function $\phi(t, x)=\mathbf{P}_{t} \varphi(x)$ can be rewritten in the form

$$
\phi(t, x)=\sup \left\{-\int_{0}^{t} H^{\star}\left(u_{s}\right) d s+\varphi\left(X_{t}^{x}(u)\right) ; u \in L^{\infty}((0,1) ; U)\right\}
$$

and hence $\phi(t, x)=\sup _{z \in \mathbf{R}^{d}}\left(\varphi(z)+\mathbf{p}_{t \mid 0}^{X}(z \mid x)\right)$, where

$$
\mathbf{p}_{t \mid 0}^{X}(z \mid x)=\sup \left\{-\int_{0}^{t} H^{\star}\left(u_{s}\right) d s ; u \in L^{\infty}((0,1) ; U): X_{t}^{x}(u)=z\right\} .
$$

If $z \notin x+t U$ we clearly have $\mathbf{p}_{t \mid 0}^{X}(z \mid x)=-\infty$. Now let us suppose that $z \in x+t U$. Letting

$$
u(s)=\frac{z-x}{t} \quad \forall 0 \leqslant s \leqslant t
$$

we obtain $X_{t}^{x}(u)=z$ and therefore

$$
\mathbf{p}_{t \mid 0}^{X}(z \mid x) \geqslant-t H^{\star}\left(\frac{z-x}{t}\right) .
$$

On the other hand, using the fact that $H^{\star}$ is convex

$$
\int_{0}^{t} H^{\star}\left(u_{s}\right) d s \geqslant t H^{\star}\left(\frac{1}{t} \int_{0}^{t} u_{s} d s\right) \geqslant t H^{\star}\left(\frac{z-x}{t}\right)
$$

as soon as $u(\cdot)$ is chosen in such a way that $X_{t}^{x}(u)=z$. This together with (8) gives

$$
\mathbf{p}_{t \mid 0}^{X}(z \mid x)= \begin{cases}-t H^{\star}\left(\frac{z-x}{t}\right) & \text { if } z \in x+t U, \\ -\infty & \text { otherwise. }\end{cases}
$$

One concludes that

$$
\mathbf{P}_{t} \varphi(x)=\sup _{z \in x+t U}\left(\varphi(z)-t H^{\star}\left(\frac{z-x}{t}\right)\right) \quad \text { (Oleinik-Lax formula) }
$$

and for any performance density $\mu$ on $\mathbf{R}^{d}$

$$
\mu \mathbf{P}_{t}(z)=\sup _{x: z-x \in t U}\left(\mu(x)-t H^{\star}\left(\frac{z-x}{t}\right)\right) .
$$

Let us remark that we can replace in the above construction the time space $(0,1)$ by the interval $(0, T), T>0$. Moreover if we choose the initial value 
$x=0$ and we define $X_{t}(u)=\int_{0}^{t} u_{s} d s$ then the performance density $\mu_{t}$ of $X_{t}$ is given by

$$
\mu_{t}(x)= \begin{cases}-t H^{\star}\left(\frac{x}{t}\right) & \text { if } x \in t U \\ -\infty & \text { otherwise. }\end{cases}
$$

Proposition 2 (Weak Law of Large Numbers). If $H^{\star}: U \longrightarrow \mathbf{R}^{+}$is a convex function and has a unique minimum $X^{\star}$ such that $H^{\star}\left(X^{\star}\right)=0$ then

$$
\frac{X_{t}}{t} \underset{t \rightarrow \infty}{\stackrel{w}{\longrightarrow}} X^{\star}
$$

in the sense that the performance measures of $X_{t} / t$ converge weakly to $\delta_{X^{\star}}$, as $t \rightarrow \infty$.

P r o of. Let $m_{t}$ be the performance density of the optimization variable $X_{t} / t$. In view of (9) we have that

$$
m_{t}(x)= \begin{cases}-t H^{\star}(x) & \text { if } x \in U, \\ -\infty & \text { otherwise }\end{cases}
$$

For any bounded and continuous test function $\varphi$ on $\mathbf{R}^{d}$ we clearly have

$$
\left\langle\varphi, m_{t}\right\rangle=\sup _{x \in U}\left(\varphi(x)-t H^{\star}(x)\right) \underset{t \rightarrow \infty}{\stackrel{w}{\longrightarrow}} \varphi\left(X^{\star}\right)=\left\langle\varphi, \delta_{X^{\star}}\right\rangle .
$$

Proposition 3 (Central Limit Theorem). Suppose the assumptions of proposition 2 hold with $X^{\star}=0$ and $H^{\star}$ is a twice differentiable function on $U$ such that

$$
\left(H^{\star}\right)^{\prime \prime}(0)>0 \text { and }\left\|H^{\star}(u)-H^{\star}(v)\right\| \leqslant C|u-v|^{\alpha}
$$

for some $C<\infty$ and $\alpha>0$. Then we have

$$
\frac{X_{t}}{\sqrt{t}} \underset{t \rightarrow \infty}{\stackrel{w}{\longrightarrow}} Z
$$

where $Z$ is a quadratic optimization variable with performance density $\nu(z)=$ $-\frac{1}{2} z^{\prime}\left(H^{\star}\right)^{\prime \prime}(0) z$.

$\mathrm{P} \mathrm{r}$ o of. Let $m_{t}$ be the performance density of the optimization variable $X_{t} / \sqrt{t}$. In view of $(9)$ we have that

$$
m_{t}(x)= \begin{cases}-t H^{\star}\left(\frac{x}{\sqrt{t}}\right) & \text { if } \frac{x}{\sqrt{t}} \in U, \\ -\infty & \text { otherwise. }\end{cases}
$$

For any bounded and continuous test function $\varphi$ with compact support $K$ on $\mathbf{R}^{d}$ we clearly have

$$
\left\langle\varphi, m_{t}\right\rangle=\sup _{x \in K}\left(\varphi(x)-t H^{\star}\left(\frac{x}{\sqrt{t}}\right)\right) .
$$


Under our assumptions for any $x$ there exists a constant $\lambda \in[0,1]$ such that

$$
H^{\star}\left(\frac{x}{\sqrt{t}}\right)=\frac{1}{2 t} x^{\prime} H^{\star}\left(\lambda \frac{x}{\sqrt{t}}\right) x .
$$

This yields that $\left\langle\varphi, m_{t}\right\rangle=\sup _{x \in K}\left(\varphi(x)-\frac{1}{2} x^{\prime} H^{\star}(\lambda x / \sqrt{t}) x\right)$ and therefore

$$
\left|\left\langle\varphi, m_{t}\right\rangle-\langle\varphi, \nu\rangle\right| \leqslant \sup _{x \in K}\left(-\frac{1}{2} x^{\prime}\left\|H^{\star}\left(\lambda \frac{x}{\sqrt{t}}\right)-H^{\star}(0)\right\| x\right) \leqslant \text { const } t^{-\alpha / 2}
$$

the end of the proof is now straightforward.

3. Applications to Mathematical Morphology. Our aim is now to discuss the connections between. Maslov's optimization theory and Mathematical Morphology. More precisely we will now show how the preceding framework provides a natural analytic basis for studying the evolution of the so-called morphological dilations. These transformations model the rate of change of a given signal under a continuous multiscale dilation (see [6], [19]). Mathematical Morphology is a particular discipline in Image Processing. In this framework the image is model as a function $\mu: \mathbf{R}^{d} \longrightarrow \mathbf{R}$. The multiscale morphological dilation of the signal $\mu$ is defined as a space-scale function which represents at each scale $t \in \mathbf{R}_{+}$the transformation of the signal by a given structuring function $g: U \rightarrow \mathbf{R}$ where $U$ is a compact subset of $\mathbf{R}^{d}$.

This transformation is defined as follows

$$
D_{t}(\mu)(x)=\sup _{z: x \rightarrow z \in t U}\left(\mu(z)-t g\left(\frac{x-z}{t}\right)\right) .
$$

When $g(U)=\{0\}$ the structuring function is said to be flat and the dilation transformation takes the form $D_{t}(\mu)(x)=\sup _{z \in t U} \mu(x-z)$. In this particular situation the transformation is called a multiscale dilation of the signal $\mu$ by the structuring element $U$.

Let us assume that $\mu$ is a performance density on $\mathbf{R}^{d}$ and $g: U \rightarrow \mathbf{R}_{+}$ is a convex structuring function. Using the preceding results one concludes that the mapping $(t, x) \rightarrow D_{t}(\mu)(x)=\mu \mathbf{P}_{t}(x)$ is a generalized solution of the Hamilton-Jacobi equation

$$
\left\{\begin{array}{l}
\frac{\partial m}{\partial t}=g^{\star}\left(-D_{x} m\right) \\
m(0, \cdot)=\mu
\end{array}\right.
$$

Workers on image processing have attempted to make sense out of the scale evolution associated with the continuous-space multiscale morphological dilation (10). We note that the idempotent analysis framework is a rather natural way to think about such transformations and characterizes their time evolution in terms of Hamilton-Jacobi equations. 
This new application of idempotent analysis also provides an alternative approach to the Beucher's morphological gradient or the sup-inf derivative notions presented in [6]. Moreover, in contrast with the classical works on the subject we do not need to assume that the compact set $U$ has smooth boundaries or that the structuring function $g$ is strictly convex and/or has an inverse gradient everywhere [31].

To make the above ideas firm let us present some structuring functions arising in this framework.

Ex a m p le 1. If $g(U)=\{0\}$ and $U=\left\{u \in \mathbf{R}^{d}:\|u\|_{\alpha} \leqslant 1\right\}$ for some $\alpha \in[1, \infty]$ then using the classical formula

$$
\sup _{\|u\|_{\alpha} \leqslant 1}\langle q, u\rangle=\|q\|_{\beta}, \quad \frac{1}{\beta}+\frac{1}{\alpha}=1
$$

we arrive at $g^{\star}(q)=\|q\|_{\beta}$ and therefore the equation (11) has the form

$$
\frac{\partial m}{\partial t}=\left\|D_{x} m\right\|_{\beta}
$$

For instance we have

1) if $\alpha=1, \beta=\infty$ then (11) takes the form $\partial m / \partial t=\bigoplus_{1 \leqslant i \leqslant d}\left|\partial m / \partial x_{i}\right|$;

2) if $\alpha=\infty, \beta=1$ then (11) takes the form $\partial m / \partial t=\sum_{1 \leqslant i \leqslant d}\left|\partial m / \partial x_{i}\right|$;

3) if $\beta \geqslant 1$ then (11) takes the form $(\partial m / \partial t)^{\beta}=\sum_{1 \leqslant i \leqslant d}\left|\partial m / \partial x_{i}\right|^{\beta}$.

$\mathrm{Example} \mathrm{2.} \mathrm{If} \mathrm{the} \mathrm{structuring} \mathrm{function} g$ is given by $g: u \in$ $U \longrightarrow g(u)=g(0)\left(1-\|u\|_{\alpha}^{\alpha}\right)^{1 / \alpha}$ where $g(0) \geqslant 0$ and $U=\left\{u \in \mathbf{R}^{d}:\|u\|_{\alpha} \leqslant 1\right\}$ for some $\alpha \in[1, \infty]$ then in the same way as before we arrive at

$$
g^{\star}(q)=\|(g(0), q)\|_{\beta}=\left(g^{\beta}(0)+\|q\|_{\beta}^{\beta}\right)^{1 / \beta}, \quad \frac{1}{\beta}+\frac{1}{\alpha}=1,
$$

and therefore the equation (11) has the form

$$
\frac{\partial m}{\partial t}=\left\|\left(g(0), D_{x} m\right)\right\|_{\beta}
$$

For instance we have

1) if $\alpha=1, \beta=\infty$ then (11) takes the form

$$
\frac{\partial m}{\partial t}=g(0) \oplus \bigoplus_{1 \leqslant i \leqslant d}\left|\frac{\partial m}{\partial x_{i}}\right| ;
$$

2) if $\alpha=\infty, \beta=1$ then (11) takes the form

$$
\frac{\partial m}{\partial t}=g(0)+\sum_{1 \leqslant i \leqslant d}\left|\frac{\partial m}{\partial x_{i}}\right|
$$


3) if $\beta \geqslant 1$ then (11) takes the form

$$
\left(\frac{\partial m}{\partial t}\right)^{\beta}=g^{\beta}(0)+\sum_{1 \leqslant i \leqslant d}\left|\frac{\partial m}{\partial x_{i}}\right|^{\beta}
$$

\section{REFERENCES}

1. Akian M., Quadrat J.P., Viot M. Bellman processes. - Lecture Notes in Control and Inform. Sci., 1994, v. 199, p. 302-311.

2. Akian M., Quadrat J.P., Viot $M$. Duality between probability and optimization. Idempotency. Ed. by J. Gunawardena. Cambridge: Cambridge Univ. Press, 1998, p. 331-353.

3. Bacelli F., Cohen G., Olsder G.J., Quadrat J.P. Synchronization and Linearity. An Algebra for Discrete Event Systems. New York: Wiley, 1992. 489 p.

4. Barles G. Solutions de viscosité des équations de Hamilton-Jacobi. Paris: SpringerVerlag, 1994. $194 \mathrm{p}$.

5. Bellman R. E., Dreyfus S. E. Dynamic Programing and Applications. Paris: Dunod, 1965.

6. Brockett $R$. $W$., Maragos $P$. Evolution equations for continuous-scale morphological filtering. - IEEE Trans. Signal Processing, 1994, v. 42, № 12.

7. Cox $H$. Estimation of state variables via dynamic programming. - Proceedings of the 1964 Joint Automatic Control Conference (Stanford, California), p. 376-381.

8. Crandall M. G., Ishii H., Lions. P. L. User's guide to viscosity solutions of second order partial differential equations. - Bull. Amer. Math. Soc., 1992, v. 27, p. 1-67.

9. Crandall M. G., Lions P.L. Viscosity solutions of Hamilton-Jacobi equations. Trans. Amer. Math. Soc., 1983, v. 277, p. 1-42.

10. Cuninghame-Green R. Minimax Algebra. Berlin-Heidelberg: Springer-Verlag, 1979.

11. Del Moral P., Doisy $M$. On the Applications of Maslov Optimization Theory. Preprint, 1998.

12. Del Moral P. Maslov optimization theory: optimality versus randomness. - Appendix in Kolokoltsov V. N., Maslov V. P. Idempotency Analysis and its Applications. Dordrecht: Kluwer, 1997.

13. Del Moral P. On the stability of Maslov optimization processes. - 34th Conference on Decision and Control, December 1995.

14. Del Moral P. Maslov optimization theory, topological aspects. - Idempotency. Ed. by J. Gunawardena. Cambridge: Cambridge Univ. Press, 1998, p. 354-382.

15. Del Moral P., Salut G. Random particle methods in (max, +)-optimization problems. - Idempotency. Ed. by J. Gunawardena. Cambridge: Cambridge Univ. Press, 1998, p. 383-391.

16. Del Moral P., Noyer J. C., Salut G. Maslov optimization theory: Stochastic interpretation, particle resolution. - Lecture Notes in Control and Inform. Sci., 1994, v. 199, p. $312-318$.

17. Del Moral P., Miclo L. On the Convergence and the Applications of the Generalized Simulated Annealing. Publications du Laboratoire de Statistiques et Probabilités. Université Paul Sabatier, 1996.

18. Дудников П.И., Самборский $C . Н$. Эндоморфизмы полумодулей над полукольцами с идемпотентной операцией. Препринт № 87-48. Киев: Ин-т матем. АН Укражны, 1987.

19. Heijmans H.J.A. The algebraic basis of mathematical morphology. I. Dilations and erosions. - Computer Vision, Graphics, and Image Processing, 1990, v. 50, p. 245295.

20. Колокольчов В.H. Полукольцевые аналоги линейных эквивалентных пространств. - Тезисы Всесоюзной школы «Оптимальное управление, геометрия и анализ», Кемерово, 1988, с. 30. 
21. Kolokoltsov V.N. Proceedings of the Idempotency workshop. Hewlett Packard Laboratories, Bristol 3-7 October 1994.

22. Колокольчов В.H., Маслов В. П. Идемпотентный анализ как аппарат теории управления и оптимального синтеза. - Функц. анализ и прилож., 1989, т. 23, № 1, c. 1-14.

23. Колокомьчов В.Н., Маслов В. П. Общий вид эндоморфизмов в пространстве непрерывных функций со значениями в числовом коммутативном полукольце (с операцией $\oplus=\max$ ). - Докл. АН СССР, 1987, т. 295, № 2, с. 283-287.

24. Litvinov G., Maslov V.P. Correspondence Principle for Idempotent Calculus and Some Computer Applications. Preprint IHES-M-95-33. Institut des Hautes Etudes Scientifiques: Bures-sur-Yrette, 1995.

25. Маслов В. П. Операционные методы. М.: Мир, 1987.

26. Maslov V. P. Quasilinear systems which are linear in some semimoduli. - Congrès international sur les problèmes hyperboliques, 13-17 Janvier 1986, Saint Etienne, France.

27. Maslov V.P., Samborskii S. N. Idempotent Analysis. Providence, RI: Amer. Math. Soc., 1992. 210 p.

28. Maslov V.P., Samborskii S. N. Stationary Hamilton-Jacobi and Bellman equations. - Idempotent Analysis. Ed. by V. P: Maslov et al. Providence, RI: Amer. Math. Soc., 1992, pp. 119-133.

29. Miclo $L$. Recuit simulé sans potentiel sur un ensemble fini. - Lecture Notes in Math., 1992, v. 1526 , p. 47-60.

30. Quadrat J.P. Théorèmes asymptotiques en programmation dynamique. C. R. Acad. Sci., Paris, 1990, v. 311, p. 745-748.

31. Van der Boomgaard R. Mathematical Morphology: Extensions towards computer vision. Ph. D. Thesis, Univ. of Amsterdam, 1992. 